\title{
Prognostic role of myoferlin expression in patients with clear cell renal cell carcinoma
}

\author{
Dae Hyun Song ${ }^{1,2,4}$, Gyung Hyuck Ko ${ }^{1,2,3}$, Jeong Hee Lee $^{1,2,3}$, Jong Sil Lee Le, $^{1,3}$, Jung \\ Wook Yang ${ }^{3}$, Min Hye Kim${ }^{3}$, Hyo Jung An', Myoung Hee Kang ${ }^{1,2,5}$, Kyung Nyeo \\ Jeon ${ }^{1,2,6}$ and Dong Chul Kim ${ }^{1,2,3}$ \\ ${ }^{1}$ Gyeongsang National University School of Medicine, Jinju, South Korea \\ ${ }^{2}$ Gyeongsang Institute of Health Science, Gyeongsang National University, Jinju, South Korea \\ ${ }^{3}$ Department of Pathology, Gyeongsang National University Hospital, Jinju, South Korea \\ ${ }^{4}$ Department of Pathology, Gyeongsang National University Changwon Hospital, Changwon, South Korea \\ ${ }^{5}$ Department of Internal Medicine, Gyeongsang National University Changwon Hospital, Changwon, South Korea \\ ${ }^{6}$ Department of Radiology, Gyeongsang National University Changwon Hospital, Changwon, South Korea \\ Correspondence to: Dong ChulKim, email: kdcjes@gmail.com \\ Keywords: clear cell renal cell carcinoma, myoferlin, prognosis, disease-free survival, immunohistochemistry \\ Received: July 04, $2017 \quad$ Accepted: August 27, $2017 \quad$ Published: October 06, 2017 \\ Copyright: Song et al. This is an open-access article distributed under the terms of the Creative Commons Attribution License 3.0 \\ (CC BY 3.0), which permits unrestricted use, distribution, and reproduction in any medium, provided the original author and source \\ are credited.
}

\section{ABSTRACT}

Objectives: In patients with cancer, myoferlin protein hyperexpression has been correlated with poor patient prognosis. Here, we evaluated myoferlin expression in patients with clear cell renal cell carcinoma (CCRCC) and investigated the prognostic significance of myoferlin expression in these patients.

Materials and Methods: One hundred and fifty-two patients with CCRCC who underwent treatment at Gyeongsang National University Hospital, Korea, between January 2000 and December 2009 were enrolled. Immunohistochemical analysis was performed on tissue microarray blocks produced from surgical specimens. Surgical specimen cancerous cells were graded as showing myoferlin hyperexpression or hypoexpression by comparison with intratumoral endothelial cells. Disease-free survival was evaluated using Kaplan-Meier analysis. Cox regression analysis was used to determine the relationships between myoferlin expression levels, risk factors, and prognosis.

Results: Seventy-one of $\mathbf{3 0 4}$ cores exhibited myoferlin hyperexpression. T stage was not associated with myoferlin hyperexpression, whereas a high Fuhrman nuclear grade was significantly associated with myoferlin hyperexpression. Kaplan-Meier analysis revealed that patients with $\mathrm{T}$ stage $>2$, Fuhrman nuclear grade $>2$, and those with myoferlin hyperexpression had poorer disease-free survival compared to those with lower $\mathrm{T}$ stage, lower Fuhrman nuclear grade, and myoferlin hypoexpression (all p <0.001). Furthermore, myoferlin hyperexpression was significantly associated with disease-free survival on Cox regression analysis (hazard ratio, $4.604 ; 95 \%$ confidence interval, 1.893-11.199; $p=0.001$ ).

Conclusion: Myoferlin expression could be a potential prognosticator in patients with CCRCC, and might be a useful marker for oncologic surveillance in such patients. 
Table 1: Clinicopathological information of 152 clear cell renal cell carcinoma(RCC) patients

\begin{tabular}{|c|c|c|c|}
\hline & Variable & & Value (percentage) \\
\hline & Patients number & & 152 \\
\hline & Cores number of TMA & & 304 \\
\hline \multirow[t]{9}{*}{ Clinical information } & Age [range] & & mean 59.9 [32-83] \\
\hline & Sex (Male/Female) & & $109 / 43$ patients \\
\hline & Advanced RCC & Lung metastasis & 9 patients \\
\hline & & Multiple metastasis & 6 patients \\
\hline & & Bone metastasis & 4 patients \\
\hline & & Brain metastasis & 2 patients \\
\hline & & Liver metastasis & 1 patients \\
\hline & & Local recurrence & 3 patients \\
\hline & Follow-up period & & Mean 4.33 years \\
\hline \multirow[t]{13}{*}{ Patologic information } & T stage & 1a & $91(59.9 \%)$ patients \\
\hline & & $1 b$ & $24(15.8 \%)$ patients \\
\hline & & $2 \mathrm{a}$ & $9(5.9 \%)$ patients \\
\hline & & $2 b$ & $3(2.0 \%)$ patients \\
\hline & & $3 a$ & $21(13.8 \%)$ patients \\
\hline & & $3 b$ & $2(1.3 \%)$ patients \\
\hline & & 4 & $2(1.3 \%)$ patients \\
\hline & Fuhrman grade & 1 & $26(17.1 \%)$ patients \\
\hline & & 2 & $102(67.1 \%)$ patients \\
\hline & & 3 & $19(12.5 \%)$ patients \\
\hline & & 4 & $5(3.3 \%)$ patients \\
\hline & Myoferlin & Hyperexpression & $71(23.4 \%)$ cores \\
\hline & & Hypoexpression ${ }^{*}$ & $233(76.6 \%)$ cores \\
\hline Total & & & $\begin{array}{c}152 \text { patients } \\
304 \text { cores }\end{array}$ \\
\hline
\end{tabular}

*, hypo-expression contains negative expression.

\section{INTRODUCTION}

Clear cell renal cell carcinoma (ccRCC) accounts for $70-80 \%$ of RCCs [1], and $\sim 20-30 \%$ of these patients progress to an advanced stage (recurrence or distant metastasis) after surgery [2]. The frequency of postoperative radiologic surveillance, which consists of chest and abdominal radiologic imaging, is determined by a patient's 5-year risk for recurrence or distant metastases $[3,4]$. The tumor-node-metastasis staging of the American Joint Committee on Cancer (AJCC) [5] and Fuhrman nuclear grade [6] are well established predictive risk factors for recurrence. However, to ensure that treatment for patients with ccRCC patients is efficacious, additional reliable prognosticators are needed.

Myoferlin protein expression has been recently associated with poor prognosis in patients with cancer [7-9]. The 6-membered Ferlin family are mammalian proteins that share homology with the Fer-1 family of Caenorhabditis Elegans [10] and include myoferlin, dysferlin, otoferlin, fer1L4, fer1L5, and fer1L6. Fer-1 defects have been associated with infertility due to the abnormal fusion of cellular membranes in developing sperm [11]. Ferlins have also been implicated in calcium- 
sensing, cellular repair, vesicle trafficking, and membrane fusion in skeletal muscle [12]. In normal embryonic development or muscle regeneration, myoferlin is highly expressed during myoblast fusion [12]. However, the physiological function(s) of myoferlin have not been fully elucidated.

Myoferlin overexpression has been noted in gastric, thyroid, breast, colorectal, hepatic, pancreatic, ovarian, cervical, and endometrial cancers [13]. Furthermore, in MDA-MB-231 human breast cancer cells, myoferlin depletion induced a mesenchymalto-epithelial transition, in vitro, and reduced tumoral proliferation and invasion, in vivo $[14,15]$. Leung et al. showed tumorigenetic role of myoferlin [16]. According to recent clinical studies, patients with pancreatic or oropharyngeal squamous cell carcinoma that showed myoferlin hyperexpression had poorer survival outcomes [9, 17].

Here, we investigated myoferlin expression in patients with ccRCC and evaluated the prognostic significance of myoferlin expression in these patients.

\section{RESULTS}

\section{Clinicopathological data}

The clinicopathological data of patients with ccRCC $(\mathrm{n}=152)$ are summarized in Table 1 . There were 115 (75.7\%), $12(7.9 \%), 23(15.1 \%)$, and $2(1.3 \%)$ patients with T stage $1,2,3$, and 4 disease, respectively. Twentyfive patients had progression of diseases, Of which nine patients exhibited metastatic ccRCC of the lung. And 6 patients showed metastasis to multiple organs, such as the lung, bone and/or brain. The majority of patients had Fuhrman nuclear grade 2 tumors. Of the 304 cores evaluated, 71 exhibited myoferlin hyperexpression (Hyper-MYOF), and 233 had myoferlin hypoexpression (Hypo-MYOF) (Figure 1A and 1B).

\section{Correlation of myoferlin expression with $\mathrm{T}$ stage and Fuhrman nuclear grade in patients with ccRCC}

The relationships between myoferlin expression and $\mathrm{T}$ stage are summarized in Table 2. There were no significant differences in the incidence of Hyper-MYFO among patients with $\mathrm{T}$ stage 1 and those with $\mathrm{T}$ stage $\geq 2$ (Chi squared, $p=0.240$ ). The relationships between myoferlin expression and Fuhrman nuclear grade are summarized in Table 3. A Fuhrman nuclear grade $\geq 3$ was significantly associated with the incidence of HyperMYOF (Chi squared, $\mathrm{p}<0.001$ ).

\section{The significance of Hyper-MYOF on survival in patients with ceRCC}

According to Kaplan-Meier analysis, patients with Hyper-MYOF, high T stage $(\geq 2)$, or high Fuhrman nuclear grade $(\geq 3)$ had poorer disease-free survival (DFS, $\mathrm{p}<0.001$ ) compared to patients with Hypo-MYOF (Figure 2A-2C). Cox proportional hazards regression analysis revealed that age $\geq 59$ years (hazard ratio [HR], 3.497; $95 \%$ confidence interval [CI], 1.372-8.915; $\mathrm{p}=0.009), \mathrm{T}$ stage $\geq 2$ (HR, 2.313; 95\% CI, 7.719-64.494; p <0.001), Fuhrman nuclear grade $\geq 3$ (HR, 2.997; 95\% CI, 1.2707.070; $\mathrm{p}=0.012$ ), and Hyper-MYOF (HR, 4.604; 95\%

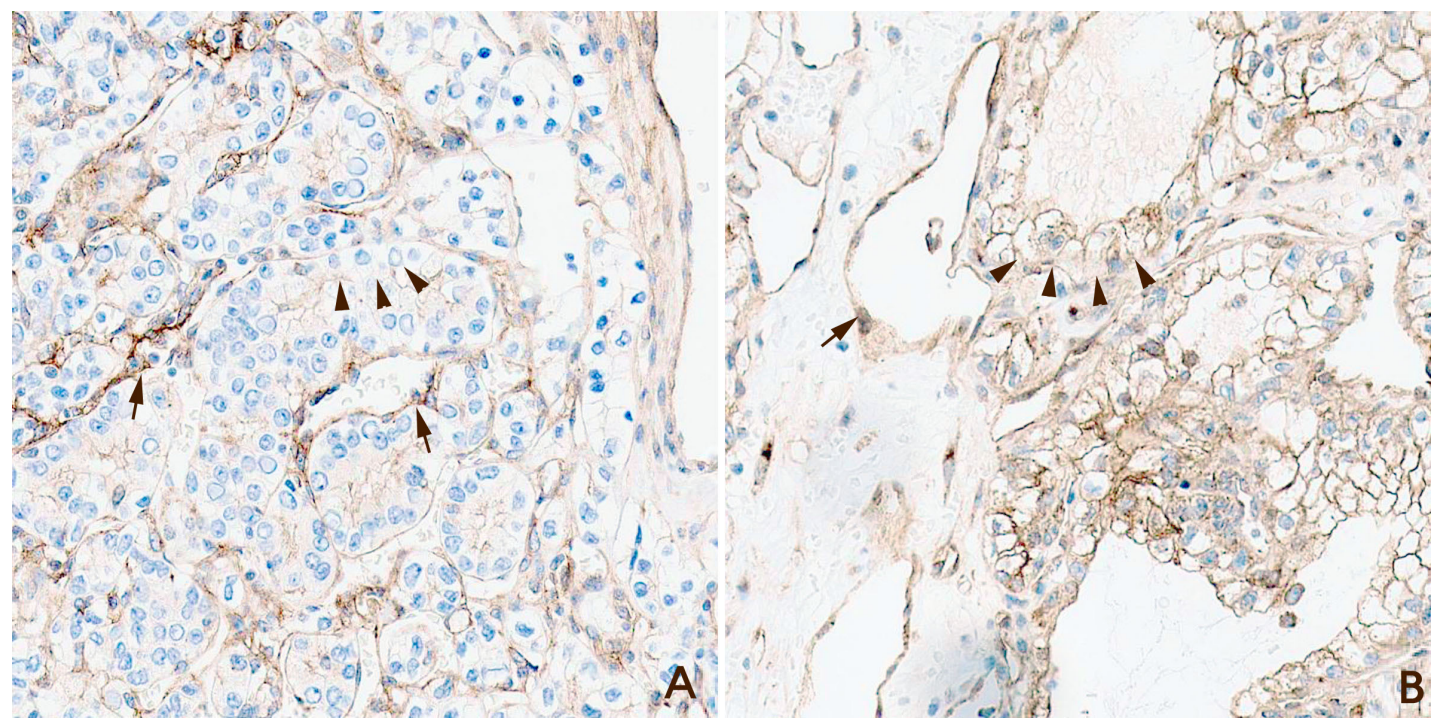

Figure 1: Examples of myoferlin staining grades. (A) Myoferlin hypoexpression. Clear cell renal cell carcinoma (ccRCC) cells revealed weaker expression compared with capillary endothelial cells (arrow, endothelial cells; arrow head, ccRCC cells; Magnification x200). (B) Myoferlin hyperexpression. Clear cell renal cell carcinoma (ccRCC) cells exhibited stronger expression compared with capillary endothelial cells (arrow, endothelial cells; arrow head, ccRCC cells; Magnification x200). 
Table 2: Correlation between myoferlin expression, $T$ stage and Fuhrman nuclear grade in clear cell renal cell carcinomas

\begin{tabular}{lccccc}
\hline & & \multicolumn{2}{c}{ Myoferlin expression } & Total & P value \\
\cline { 3 - 4 } & & Hypo $^{*}$ & Hyper & & \\
\hline T stage & 1 & 180 & 50 & 230 & $\mathrm{P}=0.240$ \\
& $\geqq 2$ & 53 & 21 & 74 & \\
Fuhrman nuclear grade & 1,2 & 205 & 50 & 255 & $\mathbf{P}<\mathbf{0 . 0 0 1}$ \\
& 3,4 & 28 & 21 & 49 & \\
Total & & 233 & 71 & 304 & \\
\hline
\end{tabular}

*, hypo-expression contains negative expression.

Table 3: Multivariate analyses of recurrent-free survival in 152 clear cell renal cell carcinoma patients

\begin{tabular}{|c|c|c|c|c|c|c|c|}
\hline \multirow{2}{*}{ Variable } & & \multicolumn{3}{|c|}{ Disease-free survival } & \multicolumn{3}{|c|}{ Disease-specific survival } \\
\hline & & HR & $95 \% \mathrm{CI}$ & $p$ value & HR & $95 \%$ CI & P value \\
\hline Age & $<59$ vs. $\geqq 59$ & 3.497 & $1.372-8.915$ & 0.009 & 0.945 & $0.453-1.972$ & 0.880 \\
\hline Sex & male vs. female & 1.339 & $0.428-4.191$ & 0.617 & 0.221 & $0.084-0.585$ & 0.002 \\
\hline T stage & 1 vs. $2 \sim 4$ & 22.313 & 7.719-64.494 & $<0.001$ & 33.420 & $10.938-102.109$ & $<0.001$ \\
\hline $\begin{array}{l}\text { Fuhrman } \\
\text { grade }\end{array}$ & 1,2 vs. 3,4 & 2.997 & $1.270-7.070$ & 0.012 & 4.009 & $1.694-9.487$ & 0.002 \\
\hline Myoferlin & hypo vs. hyper & 4.604 & $1.893-11.199$ & 0.001 & 1.240 & $0.490-3.135$ & 0.650 \\
\hline
\end{tabular}

CI, 1.893-11.199; $\mathrm{p}<0.001)$ were significantly associated with DFS (Table 3). Hyper-MYOF was not significantly associated with disease-specific survival (DSS, $p=0.650$; Table 4).

\section{DISCUSSION}

In the present study, ccRCC patients with HyperMYOF had significant poorer DFS compared to those with Hypo-MYOF. However, Hyper-MYOF was not associated with DSS in patients with ccRCC. This is the first study to demonstrate an association between high myoferlin expression and prognosis in patients with ccRCC. Furthermore, a high Fuhrman grade was associated with Hyper-MYOF, whereas $T$ stage was not associated with myoferlin hyperexpression.

Here, we found that myoferlin might be a valuable prognostic marker in patients with ccRCC. Particularly, Hyper-MYOF had greater significance than the Fuhrman nuclear grade at predicting DFS. Furthermore, although, $\mathrm{T}$ stage is considered a valuable prognostic factor, in the present study, 5 patients with advanced T1 stage
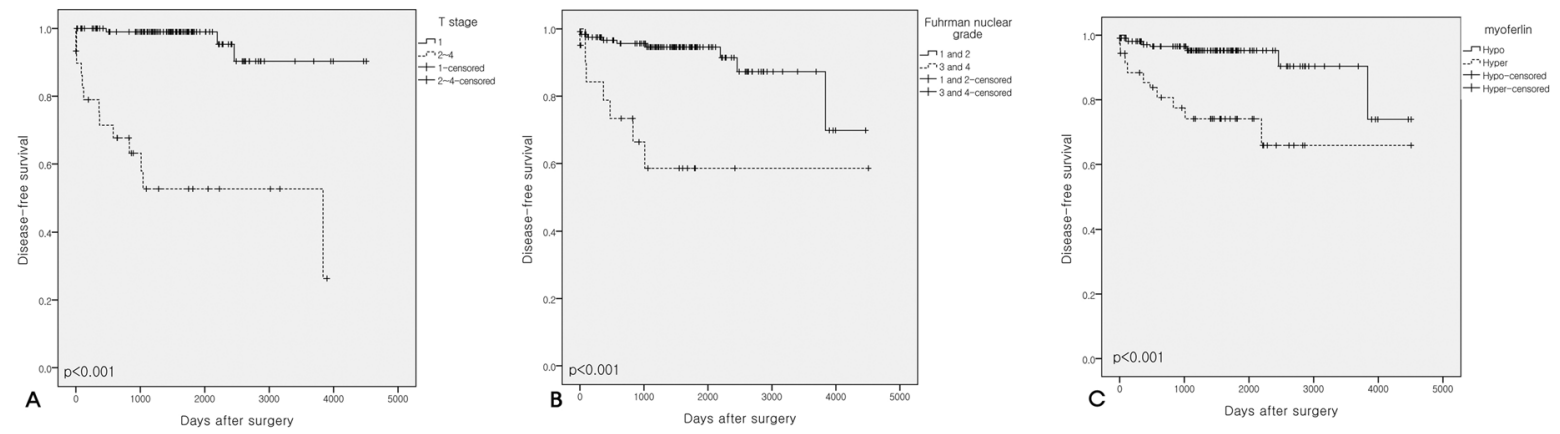

Figure 2: Kaplan-Meier survival curves. (A) Disease-free survival according to $\mathrm{T}$ stage. (B) Disease-free survival according to Fuhrman nuclear grade. (C) Disease-free survival according to myoferlin expression levels. High T stage, high Fuhrman nuclear grade, and myoferlin hyperexpression were all significantly associated with poorer disease-free survival. 
Table 4: Clinicopathological information of advanced renal cell carcinoma patients with $\mathrm{T} 1$ stage tumor

\begin{tabular}{lcccccc}
\hline Patient number & Sex/age & T stage & $\begin{array}{c}\text { Furhman nuclear } \\
\text { grade }\end{array}$ & $\begin{array}{c}\text { Myoferlin } \\
\text { expression }\end{array}$ & $\begin{array}{c}\text { Follow up duration to } \\
\text { recurrence (years) }\end{array}$ & Recurrence \\
\hline No.1 & M/64 & $1 \mathrm{a}$ & 2 & Hyper & 6 & Local recurrence \\
No.2 & M/67 & $1 \mathrm{a}$ & 3 & Hyper & 3.8 & Lung meta* \\
No.3 & M/71 & $1 \mathrm{~b}$ & 3 & Hypo & 7.6 & Lung meta \\
No.4 & $\mathrm{F} / 78$ & $1 \mathrm{a}$ & 3 & Hypo & 3.4 & Bone meta \\
No.5 & $\mathrm{M} / 72$ & $1 \mathrm{a}$ & 1 & Hypo & 6.8 & Bone meta \\
\hline
\end{tabular}

*, meta means metastasis

primary ccRCC, of whom 3 had Fuhrman nuclear grade 3 (high) and 2 had Fuhrman nuclear grade 2 (low), also had Hyper-MYOF (Table 4). Therefore, we suggest that even in patients with a low T stage, a high Fuhrman grade or Hyper-MYOF could indicate those that should be followed-up more carefully.

There are some limitations to the present study. For example, Hyper-MYOF was not associated with DSS. The lack of effect on DSS could be because of biases. For example, patients with advanced ccRCC are treated using different therapies such as the tyrosine kinase inhibitors (e.g., sunitinib or pazopanib) and inhibitors of the mammalian target of rapamycin. In addition, the initiation of treatment varied among the patients. Such differences could confound the data. Therefore, clinical evaluations considering such factors are needed to validate the significance of myoferlin expression on prognosis.

In conclusion, myoferlin overexpression had an adverse prognostic role in patients with ccRCC and might be a valuable biomarker for ccRCC oncologic surveillance. Future studies to validate the role of myoferlin in the prognosis of such patients are warranted.

\section{MATERIALS AND METHODS}

\section{Patients}

The clinicopathological data of patients with ccRCC patients who underwent treatment at Gyeongsang National University Hospital, Jinju, Korea between January 2000 and December 2009 were obtained by reviewing clinical electronic charts, retrospectively. Onehundred and fifty-two patients with ccRCC were enrolled. All patients had undergone nephrectomy. The diagnosis was histopathologically confirmed by 2 experienced pathologists. All tumors were staged using the AJCC $7^{\text {th }}$ system [5].

DFS was defined as the duration from the date of surgery to the date of tumor recurrence. DSS was defined as the duration from the date of surgery to date of death, which was mostly due to ccRCC. Advanced ccRCC with recurrence was diagnosed histopathologically via surgical biopsy or by radiography. A radiological diagnosis of lung metastasis was made when solitary or multiple spherical nodule(s) could be seen on chest computed tomography (CT) scans. Multiple lung metastases were variable in size and distributed throughout the pulmonary lobes. A radiological diagnosis of bone metastasis was made when solid enhancing masses or bone destruction became newly apparent on CT scans during follow-up. In all patients with radiologically suspected metastatic lesions, a final diagnosis was confirmed via CT-guided percutaneous biopsy and/or by combined positron emission tomography/ noncontrast CT or scintigraphy [18, 19]. This study was approved by the Institutional Review Board of Gyeongsang National University Hospital (GNUH2015-12-001) and was conducted in accordance with the principles embodied in the Declaration of Helsinki.

\section{Tissue microarray}

Nephrectomy specimens were fixed in buffered neutral formalin $(20 \%)$ overnight. Surgical samples were examined grossly and then embedded in paraffin blocks. Two representative glass slides from matched paraffin blocks were selected under microscopic review (Nikon Eclipse Ni). Two 2-mm tissue cores were obtained from each representative paraffin block that has representative Fuhrman nuclear grading at intratumoral area. and transplanted to new recipient tissue microarray blocks.

\section{Immunohistochemical analysis}

Immunohistochemical staining was performed using a primary myoferlin antibody (1:100 dilution; 7D6, Abcam, UK) as described previously [7]. The endothelial cells of the intratumoral vessels were used as a positive control. Slides from tissue microarray cores were graded by two experienced pathologists. If ccRCC cells exhibited stronger myoferlin expression compared with the membrane or cytoplasm of the endothelial cells then staining was graded as Hyper-MYOF. Similarly, if myoferlin expression was weaker than that of endothelial cells staining was graded as Hypo-MYOF. Negative 
expressed cases were contatined in Hypo-MYOF group. If the tumor cells showed heterogeneity of myoferlin in a core, representative value of the core was decided as a value of majority of tumor cells (more than 50\%). Myoferlin expression of endothelial cells was reported in previous study [20]. And intratumoral endothelial cells were used as control of myoferlin expression.

\section{Statistical analyses}

Correlations between variables were determined using the Chi squared test. Cumulative survival times were evaluated using the Kaplan-Meier method and the logrank test. Multivariate analysis using the Cox proportional hazards regression model was conducted to compare variables. A p-value of $<0.05$ was considered statistically significant. SPSS ver. 24.0 (SPSS Inc., Chicago, IL, USA) was used for all statistical analyses.

\section{Author contributions}

First author: Dae Hyun Song

Correspnding author: Dong Chul Kim

Mid author: Gyung Hyuck Ko, Jeong Hee Lee, Jong Sil Lee, Jung Wook Yang, Min Hye Kim, Hyo Jung An, Myoung Hee Kang, Kyung Nyeo Jeon.

\section{CONFLICTS OF INTEREST}

The authors declare no conflicts of interest.

\section{FUNDING}

This work was supported by biomedical research institute fund (GNUHBIF-2015-0002) from the Gyeongsang National University Hospital.

\section{REFERENCES}

1. Escudier B, Porta C, Schmidinger M, Algaba F, Patard JJ, Khoo V, Eisen T, Horwich A. Renal cell carcinoma: ESMO Clinical Practice Guidelines for diagnosis, treatment and follow-up. Ann Oncol. 2014; 25:iii49-56.

2. Stewart GD, O’Mahony FC, Powles T, Riddick AC, Harrison DJ, Faratian D. What can molecular pathology contribute to the management of renal cell carcinoma? Nat Rev Urol. 2011; 8:255-65.

3. Stewart-Merrill SB, Thompson RH, Boorjian SA, Psutka SP, Lohse CM, Cheville JC, Leibovich BC, Frank I. Oncologic surveillance after surgical resection for renal cell carcinoma: a novel risk-based approach. J Clin Oncol. 2015; 33:4151-7.

4. Kim EH, Strope SA. Postoperative surveillance imaging for patients undergoing nephrectomy for renal cell carcinoma. Urol Oncol. 2015; 33:499-502.
5. Martinez-Salamanca JI, Huang WC, Millan I, Bertini R, Bianco FJ, Carballido JA, Ciancio G, Hernandez C, Herranz F, Haferkamp A, Hohenfellner M, Hu B, Koppie T, et al. Prognostic impact of the 2009 UICC/AJCC TNM staging system for renal cell carcinoma with venous extension. Eur Urol. 2011; 59:120-7.

6. Smith ZL, Pietzak EJ, Meise CK, Van Arsdalen K, Wein AJ, Malkowicz SB, Guzzo TJ. Simplification of the Fuhrman grading system for renal cell carcinoma. Can J Urol. 2015; 22:8069-73.

7. Song DH, Ko GH, Lee JH, Lee JS, Lee GW, Kim HC, Yang JW, Heo RW, Roh GS, Han SY, Kim DC. Myoferlin expression in non-small cell lung cancer: Prognostic role and correlation with VEGFR-2 expression. Oncol Lett. 2016; 11:998-1006.

8. Blomme A, Costanza B, de Tullio P, Thiry M, Van Simaeys G, Boutry S, Doumont G, Di Valentin E, Hirano T, Yokobori T, Gofflot S, Peulen O, Bellahcene A, et al. Myoferlin regulates cellular lipid metabolism and promotes metastases in triple-negative breast cancer. Oncogene. 2017; 36:2116-30.

9. Kumar B, Brown NV, Swanson BJ, Schmitt AC, Old M, Ozer E, Agrawal A, Schuller DE, Teknos TN, Kumar P. High expression of myoferlin is associated with poor outcome in oropharyngeal squamous cell carcinoma patients and is inversely associated with HPV-status. Oncotarget. 2016; 7:18665-77. https://doi.org/10.18632/ oncotarget.7625.

10. Achanzar WE, Ward S. A nematode gene required for sperm vesicle fusion. J Cell Sci. 1997; 110:1073-81.

11. Ward S, Argon Y, Nelson GA. Sperm morphogenesis in wild-type and fertilization-defective mutants of Caenorhabditis elegans. J Cell Biol. 1981; 91:26-44.

12. Doherty KR, Cave A, Davis DB, Delmonte AJ, Posey A, Earley JU, Hadhazy M, McNally EM. Normal myoblast fusion requires myoferlin. Development. 2005; 132:5565-75.

13. Eisenberg MC, Kim Y, Li R, Ackerman WE, Kniss DA, Friedman A. Mechanistic modeling of the effects of myoferlin on tumor cell invasion. Proc Natl Acad Sci U S A. 2011; 108:20078-83.

14. Li R, Ackerman WE, Mihai C, Volakis LI, Ghadiali S, Kniss DA. Myoferlin depletion in breast cancer cells promotes mesenchymal to epithelial shape change and stalls invasion. PLoS One. 2012; 7 :e39766.

15. Volakis LI, Li R, Ackerman WE, Mihai C, Bechel M, Summerfield TL, Ahn CS, Powell HM, Zielinski R, Rosol TJ, Ghadiali SN, Kniss DA. Loss of myoferlin redirects breast cancer cell motility towards collective migration. PLoS One. 2014; 9:e86110.

16. Leung $\mathrm{C}, \mathrm{Yu} \mathrm{C}$, Lin MI, Tognon $\mathrm{C}$, Bernatchez $\mathrm{P}$. Expression of myoferlin in human and murine carcinoma tumors: role in membrane repair, cell proliferation, and tumorigenesis. Am J Pathol. 2013; 182:1900-9. 
17. Wang WS, Liu XH, Liu LX, Lou WH, Jin DY, Yang PY, Wang XL. iTRAQ-based quantitative proteomics reveals myoferlin as a novel prognostic predictor in pancreatic adenocarcinoma. J Proteomics. 2013; 91:453-65.

18. Davis SD. CT evaluation for pulmonary metastases in patients with extrathoracic malignancy. Radiology. 1991; 180:1-12.
19. Chae EJ, Kim JK, Kim SH, Bae SJ, Cho KS. Renal cell carcinoma: analysis of postoperative recurrence patterns. Radiology. 2005; 234:189-96.

20. Bernatchez PN, Sharma A, Kodaman P, Sessa WC. Myoferlin is critical for endocytosis in endothelial cells. Am J Physiol Cell Physiol. 2009; 297:C484-492. 